\title{
Caracterização dos acidentes de trânsito atendidos pelo SAMU no município de Dourados, Estado de Mato Grosso do Sul (MS), Brasil
}

\author{
Characterization of traffic accidents attended by SAMU in the city of Dourados, State of Mato \\ Grosso do Sul (MS), Brazil \\ Caracterización de acidentes de tráfico atendidos por SAMU em la ciudad de Dourados, Estado de \\ Mato Grosso do Sul (MS), Brasil
}

Recebido: 01/10/2021 | Revisado: 08/10/2021 | Aceito: 12/10/2021 | Publicado: 14/10/2021

\author{
Nilton Fernando de Lima \\ ORCID: https://orcid.org/0000-0002-6445-0399 \\ Universidade Federal da Grande Dourados, Brasil \\ E-mail: niltonfernandodelima@gmail.com \\ Ingrid Alves Rottava \\ ORCID: https://orcid.org/0000-0003-0134-8116 \\ Universidade Federal da Grande Dourados, Brasil \\ E-mail: ingrid_rottava@hotmail.com \\ Victor Jorge Guerreiro \\ ORCID: https://orcid.org/0000-0001-7924-1197 \\ Universidade Federal da Grande Dourados, Brasil \\ E-mail: victorjorgeguerreiro@hormail.com
}

\begin{abstract}
Resumo
Conhecer a epidemiologia dos acidentes de transportes terrestres é indispensável para propor ações de prevenção desse incidente voltadas para o público mais vulnerável, com isso o objetivo desse artigo foi descrever e identificar as vítimas desse agravo atendidas pelo Serviço de Atendimento Móvel de Urgência (SAMU) em Dourados, Mato Grosso do Sul. O estudo foi baseado na análise das fichas de atendimentos realizados pelo SAMU no período de 1 de junho de 2018 a 30 de maio de 2019. Os acidentes de trânsito representaram 52,77\% do total de atendimentos do SAMU, sendo que a maioria foi atendida pela unidade de suporte básico. O sexo mais acometido foi o masculino e a faixa etária mais prevalente foi de 20 a 39 anos com 53,39\% do total das vítimas. O acidente mais prevalente foi o de colisão entre veículos automotores, sendo o domingo o dia da semana de maior risco e o horário com maior registro de incidentes foi das 18:00h às 23:59h. Do total dos acidentes estudados, 12 resultaram em óbitos, sendo a maior porcentagem dos falecidos do sexo masculino $(91,66 \%)$. Dessa maneira, os formulários de atendimento do SAMU figuram-se como valorosa fonte de informação para a vigilância em saúde e demonstram sua capacidade em contribuir no acompanhamento desses agravos e apontar os fatores de risco associados aos atendimentos no município.
\end{abstract}

Palavras-chave: Acidentes de Trânsito; Serviços Médicos de Emergência; Emergências; Ferimentos e Lesões.

\begin{abstract}
Knowing the epidemiology of land transport accidents is essential to propose actions for the prevention of this incident aimed at the most vulnerable public. Thus, the objective of this article was to describe and identify the victims of this condition served by the Emergency Mobile Care Service in Dourados, Mato Grosso do Sul. The study was based on the analysis of the attendance records carried out by SAMU from June 1, 2018 to May 30, 2019. Traffic accidents represented $52.77 \%$ of the total attendances SAMU, most of which were attended by the basic support unit. The sex most affected was male and the most prevalent age group was 20 to 39 years old with $53.39 \%$ of the total victims. The most prevalent accident was the collision between motor vehicles, with Sunday being the day of the week with the highest risk and the time with the highest record of incidents was from 18:00 to 23:59. Of the total accidents studied, 12 resulted in deaths, with the highest percentage of deceased males $(91.66 \%)$. In this way, SAMU service forms are a valuable source of information for health surveillance and demonstrate their ability to contribute to the monitoring of these diseases and to point out the risk factors associated with care in the municipality.
\end{abstract}

Keywords: Traffic Accidents; Emergency Medical Services; Emergencies; Wounds and Injuries.

\section{Resumen}

Conocer la epidemiología de los accidentes de transporte terrestre es fundamental para proponer acciones de prevención de este incidente dirigidas a la población más vulnerable, con esto el objetivo de este artículo fue describir e identificar a las víctimas de esta problemática atendidas por el Servicio Móvil de Atención de Emergencias en 
Dourados, MS. El estudio se basó en el análisis de los registros de atención brindados por SAMU en el período comprendido entre junio de 2018 y mayo de 2019. Los accidentes de tránsito representaron el 52,77\% del total de visitas SAMU, la mayoría de los cuales fueron atendidos por la unidad básica de apoyo. El sexo más afectado fue el masculino y el grupo de edad más prevalente fue el de 20 a 39 años, con el 53,39\% del total de víctimas. El accidente más prevalente fue la colisión entre vehículos de motor, siendo el domingo el día de la semana con mayor riesgo y el horario con mayor registro de incidencias fue de 18: $00 \mathrm{~h}$ a 23: $59 \mathrm{~h}$. Del total de accidentes estudiados, 12 resultaron en muertes, con el mayor porcentaje de hombres fallecidos $(91,66 \%)$. Así, los formularios de atención del SAMU son una valiosa fuente de información para la vigilancia de la salud y demuestran su capacidad para contribuir al seguimiento de estos problemas de salud y señalar los factores de riesgo asociados a la atención en la ciudad.

Palabras clave: Accidentes de Tránsito; Auxiliares de Urgencia; Urgencias Médicas; Heridas y Lesiones.

\section{Introdução}

Nas primeiras quatro décadas de vida o trauma é a principal causa de óbito e representa um enorme desafio ao Brasil em termos econômicos e sociais. Segundo o Departamento de Informática do Sistema Único de Saúde (DATASUS) em 2018, das 1.316.719 mortes, 150.814 foram por causa externa e dessas 31.816 aconteceram por acidentes de trânsito (SBAIT, 2005; Brasil, 2018).

Os Acidentes de Transporte Terrestre (ATT) alcançam o mundo todo e são importantes devido a sua relevância frente ao número de mortalidade e a quantidade de pessoas portadoras de sequelas decorrentes desses. Para visualizar isso no âmbito social, analisa-se o índice APVP que são os Anos Potenciais de Vida Perdidos, no qual houve aumento de 30\% nos últimos anos em relação à acidentes e violências, enquanto que por causas naturais esse índice encontra-se em queda (Cabral, Souza \& Lima, 2011; Tobase et al., 2012).

Segundo estudos realizados pelo Instituto de Pesquisas Econômicas Aplicadas os custos anuais dos acidentes de trânsito nas cidades brasileiras se encontraram na faixa de R \$ 9,9 bilhões a R \$ 12.9 bilhões em 2014. Estudo anterior de 2003 apresentou o resultado de R\$ 5,3 bilhões por ano para todas as áreas urbanas. Em média, cada acidente custou à sociedade brasileira R \$ 261.689, sendo que um acidente envolvendo vítima fatal teve um custo médio de R\$ 664.821 (IPEA, 2020).

Soma-se a isso que, com base em dados levantados pela Organização Mundial de Saúde (OMS), mais da metade do número de mortes causadas pelo trânsito ficam entre os usuários mais vulneráveis da rodovia que são pedestres, ciclistas e motociclistas, em especial aqueles que vivem em países em desenvolvimento, como o Brasil. Dessa maneira, a maior consequência por parte dos ATT situa-se no setor de saúde, uma vez que ele é o responsável por cuidar dos enfermos, somar mortes e lidar com as sequelas dos vitimados (WHO, 2018).

Segundo o Ministério da Saúde, a implantação da Política Nacional de Atenção às Urgências, em 2003, surgiu como forma de consolidar o Sistema Único de Saúde (SUS) e suas diretrizes éticas de universalidade, integralidade e equidade, servindo também para reafirmar o compromisso das diretrizes organizacionais de descentralização, participação social e regionalização. Com isso, busca-se organizar a rede de atenção e estruturar os serviços de saúde, tornando o SAMU um bom indicador do sistema de saúde brasileiro, sendo fundamental a discussão e análise de seus indicadores (Brasil, 2006; Silva \& Nogueira, 2012).

Na cidade de Dourados, município do interior de Mato Grosso do Sul, o SAMU foi inaugurado no ano de 2008. Ele conta com 1 unidade de suporte avançado de vida, 2 unidades de suporte básico e 2 motolâncias. A central de regulação, sob gestão do município de Dourados, regula quatro cidades: Dourados, Naviraí, Nova Andradina e Ponta Porã, com uma cobertura populacional de 413.474 cidadãos, o que corresponde a 15,24\% da população total do estado (Brasil, 2006; SES-MS, 2019).

Diante do exposto, o objetivo do presente artigo é caracterizar e descrever as ocorrências dos acidentes de transporte terrestre, discutindo sua natureza, o perfil das vítimas e identificar os horários com maior prevalência de acidentes, a partir de 
análises das ocorrências atendidas pelo SAMU em Dourados, Estado de Mato Grosso do Sul, possibilitando uma observação sobre o potencial dessa fonte para os ATT em nível local.

\section{Metodologia}

Trata-se de um estudo retrospectivo descritivo, de abordagem quantitativa acerca dos acidentes de trânsito terrestre, no qual a fonte dos dados foi o Serviço de Atendimento Móvel de Urgência - SAMU, do município de Dourados - MS, referentes ao período de 1 de junho de 2018 a 30 de maio de 2019.

A coleta dos dados foi realizada em setembro de 2020, na Central de Regulação de Urgências do SAMU, onde se encontra instalado o sistema eletrônico de prontuários e atendimentos. A população amostrada foi constituída por vítimas de acidentes de trânsito terrestre, atendidas pelo SAMU na cidade de Dourados, no período proposto.

A amostra foi formada a partir dos seguintes critérios de inclusão: ocorrências com a designação inicial de acidente de trânsito, localizadas no município de Dourados - MS, no período estabelecido; e critérios de exclusão: prontuários duplicados, prontuários sem o preenchimento de duas ou mais variáveis eleitas para o estudo e/ou preenchidas incorretamente e ocorrências que evoluíram para uma designação diferente de acidente de trânsito. Após aplicação dos critérios de inclusão e exclusão, a amostra foi constituída por 1.912 vítimas de acidente de trânsito.

O local do estudo foi o município de Dourados, na região sul do Estado de Mato Grosso do Sul. No período analisado, a população do município era de 218.069 habitantes, estimada através de projeções intercensitárias do IBGE - Instituto Brasileiro de Geografia e Estatística, distribuída em um território de 4.086,38 $\mathrm{km}^{2}$ e densidade demográfica de $54,55 \mathrm{hab} / \mathrm{km}^{2}$ (SES-MS, 2019; Dourados, 2020).

As variáveis eleitas para a pesquisa foram: identificação da vítima (sexo e idade), meio de locomoção da vítima, natureza do acidente, período do dia, dia da semana, desfecho do atendimento e unidade deslocada pela central para o atendimento.

Os dados foram dispostos em planilha no Microsoft Excel 2016® elaborada para a presente análise, sem necessidade de pré-teste, ponderando-se que o prontuário do SAMU é padronizado. Realizou-se uma estatística descritiva dos dados com análise de frequência e porcentagem para as variáveis quantitativas, bem como foram calculadas as taxas de incidência considerando a população residente (taxa de incidência: TI; número de ocorrências/população exposta a cada 10.000 habitantes). Os resultados foram expostos na forma de estatística descritiva e organizados em tabelas.

A coleta e análise dos dados foram autorizadas pela Comissão de Pesquisa da Secretaria Municipal de Saúde do Município de Dourados - MS. O projeto foi submetido ao Comitê de Ética em Pesquisa envolvendo Seres Humanos da Universidade Federal da Grande Dourados - UFGD e obteve o parecer de aprovação em 2 de setembro de 2020, sob registro CAEE n 32933720.2.0000.5160.

\section{Resultados}

No período definido para o estudo, foram atendidas 1.912 vítimas de acidente de trânsito terrestre pelo SAMU na cidade de Dourados. Este valor corresponde a $52,77 \%$ do total de atendimentos realizados pelo serviço $(\mathrm{n}=3.623)$, considerando todas as causas traumáticas, apontando uma média de 159,3 acidentes por mês, com média de 5,23 acidentes por dia.

As vítimas eram predominantemente do sexo masculino, representando $57,88 \%$ do total, o que resultou em uma razão de 1,38 homens para cada mulher. A faixa etária mais frequente foi de 20 a 39 anos e a de menor frequência de 0 a 9 anos, com respectivamente $53,39 \%$ e 3,46\% do total. Das unidades de suporte, a que mais realizou atendimentos foi a de suporte básico, 
sendo a maior parte das vítimas encaminhadas para as unidades da rede pública do município. A Tabela 1 detalha cada variável analisada com seu número absoluto e porcentagem em relação ao total de atendimentos

Tabela 1. Características das ocorrências atendidas pelo SAMU. Dourados/MS, jun/2018 - maio/2019.

\begin{tabular}{|c|c|c|}
\hline Variável & n (1.912) & $\%$ \\
\hline \multicolumn{3}{|l|}{ Sexo } \\
\hline Masculino & 1107 & 57,88 \\
\hline Feminino & 800 & 41,86 \\
\hline Não informado & 5 & 0,26 \\
\hline \multicolumn{3}{|l|}{ Faixa Etária (em anos) } \\
\hline 0 a 9 & 66 & 3,46 \\
\hline 10 a 19 & 217 & 11,34 \\
\hline 20 a 39 & 1021 & 53,39 \\
\hline 40 a 59 & 418 & 21,86 \\
\hline 60 ou mais & 100 & 5,23 \\
\hline Não informado & 90 & 4,72 \\
\hline \multicolumn{3}{|c|}{ Unidade SAMU empregada no atendimento } \\
\hline Unidade de Suporte Básico - USB & 1428 & 78,73 \\
\hline Unidade de Suporte Avançado - USA & 106 & 5,85 \\
\hline Transporte & 78 & 4,29 \\
\hline Equipe Motolância & 77 & 4,25 \\
\hline Outros* & 108 & 5,95 \\
\hline Não informado & 17 & 0,93 \\
\hline \multicolumn{3}{|l|}{ Desfecho atendimento } \\
\hline Unidade de Saúde Pública & 1298 & 67,88 \\
\hline Unidade de Saúde Privada & 243 & 12,71 \\
\hline Recusa de remoção & 158 & 8,26 \\
\hline Liberação no local & 51 & 2,66 \\
\hline Evasão do local & 25 & 1,31 \\
\hline Orientação via telefone & 21 & 1,09 \\
\hline Óbito no local & 12 & 0,63 \\
\hline Outros & 87 & 4,58 \\
\hline Ignorado & 17 & 0,88 \\
\hline
\end{tabular}




\begin{tabular}{lcc}
\hline Meio de locomoção da vítima & & \\
\hline A pé & 306 & 6,21 \\
\hline Bicicleta & 1272 & 16,01 \\
\hline Motocicleta & 207 & 66,53 \\
\hline Automóvel & 8 & 10,82 \\
\hline Não informado & & 0,43 \\
\hline Natureza do acidente & 882 & 46,12 \\
\hline Colisão x motocicleta & 365 & 19,08 \\
\hline Queda ao solo em motocicleta & 161 & 8,42 \\
\hline Queda ao solo em bicicleta & 153 & 8,00 \\
\hline Colisão x automóvel & 138 & 7,21 \\
\hline Colisão x bicicleta & 119 & 6,22 \\
\hline Atropelamento & 62 & 3,24 \\
\hline Colisão x objeto fixo & 24 & 1,26 \\
\hline Capotamento de automóvel & 8 & 0,42 \\
\hline Ignorado & & \\
\hline
\end{tabular}

Legenda: $\mathrm{n}$ - total de ocorrências; \% - porcentagem do total de ocorrências; * - corpo de bombeiros militar, ambulâncias do município, remoção por terceiros. Fonte: elaborado pelo autor.

Foram contabilizados pelo SAMU, no período estudado, doze óbitos por acidente de trânsito, desses, onze vítimas eram do sexo masculino $(91,66 \%)$ e em relação à faixa etária dez encontram-se entre 20 a 39 anos e as outras duas vítimas nas faixas de 40 a 59 e 60 anos ou mais. No que diz respeito ao meio de locomoção, oito vítimas estavam em motocicletas, duas a pé, uma em automóvel e outra em bicicleta.

No total de casos analisados, ao considerar a natureza do acidente, colisão entre veículos automotores foi a razão do incidente em $65 \%$ deles. No que se refere ao meio de locomoção da vítima, as motocicletas representaram a maioria entre os veículos envolvidos em acidente de trânsito, estando presentes em 66,53\% dos incidentes. (Tabela 1).

A Tabela 2 mostra a distribuição de atendimentos por dia da semana e considerando o dia de referência a terça-feira, constatou-se que o domingo apresentou um risco 55\% maior de ocorrência de acidente de trânsito urbano, sendo os finais de semana (sábado e domingo) responsáveis por 35,77\% dos dias dos incidentes. Quanto aos períodos de risco, os chamados aconteceram cerca de 3 vezes mais das 18:00h às 23:59h em relação ao horário de referência que abrange da 00:00h às 05:59h. 
Tabela 2. Distribuição temporal dos acidentes de trânsito realizados pelo SAMU. Dourados/MS, junho/2018 - maio/2019.

\begin{tabular}{|c|c|c|c|}
\hline Variável & n (1.912) & $\%$ & TI \\
\hline \multicolumn{4}{|l|}{ Horário } \\
\hline $00: 00 h$ às $5: 59 h$ & 215 & 11,24 & 9,64 \\
\hline 06:00h às 11:59h & 496 & 25,96 & 22,24 \\
\hline $12: 00 h$ às $17: 59 h$ & 597 & 31,22 & 26,77 \\
\hline $18: 00 h$ às $23: 59 h$ & 604 & 31,58 & 27,09 \\
\hline \multicolumn{4}{|l|}{ Dia da Semana } \\
\hline Segunda - Feira & 246 & 12,87 & 11,03 \\
\hline Terça-feira & 206 & 10,77 & 9,23 \\
\hline Quarta-Feira & 241 & 12,60 & 10,80 \\
\hline Quinta-Feira & 235 & 12,29 & 10,54 \\
\hline Sexta-Feira & 300 & 15,70 & 13,45 \\
\hline Sábado & 364 & 19,04 & 16,32 \\
\hline Domingo & 320 & 16,73 & 14,35 \\
\hline
\end{tabular}

Legenda: total de ocorrências; \% - porcentagem do total de ocorrências; TI - taxa de incidência (número de ocorrências/população exposta a cada 10.000 habitantes). Fonte: Elaborada pelo autor.

A partir da análise da tabela 3, pode-se observar a associação entre sexo, faixa etária e meios de locomoção, os quais englobam as bicicletas, motocicletas, a pé e outros veículos, predominou o sexo masculino entre as vítimas e, apenas quando o meio eram os automóveis, houve destaque do sexo feminino. Quanto à faixa etária, de 0 a 9 anos ocorreu maior incidência dos acidentes em que a vítima estava de bicicleta e no restante das diferentes faixas etárias, predominaram os acidentes em motocicleta.

Tabela 3 - Caracterização das vítimas dos acidentes de trânsito realizados pelo SAMU. Dourados/MS, junho/2018 - maio/2019.

\begin{tabular}{|c|c|c|c|c|c|c|c|c|c|}
\hline \multicolumn{10}{|l|}{ Variável } \\
\hline \multirow[t]{2}{*}{ Sexo } & \multicolumn{2}{|c|}{ Masculino } & \multicolumn{2}{|c|}{ Feminino } & \multicolumn{2}{|c|}{ Ignorado } & \multicolumn{2}{|c|}{ Total } & \multirow{2}{*}{$\begin{array}{c}\text { M:F } \\
-\end{array}$} \\
\hline & $\mathrm{n}$ & $\%$ & $\mathrm{n}$ & $\%$ & $\mathrm{n}$ & $\%$ & $\mathrm{n}$ & $\%$ & \\
\hline \multicolumn{10}{|c|}{ Meio de locomoção vítima } \\
\hline A pé & 73 & 3,81 & 46 & 2,43 & 0 & 0 & 119 & 6,24 & $1,58: 1$ \\
\hline Bicicleta & 207 & 10,82 & 99 & 5,19 & 0 & 0 & 306 & 15,99 & 2,09:1 \\
\hline Motocicleta & 728 & 38,07 & 543 & 28,39 & 1 & 0,05 & 1272 & 66,52 & $1,34: 1$ \\
\hline Automóvel & 96 & 5,02 & 110 & 5,76 & 1 & 0,05 & 207 & 10,83 & $0,87: 1$ \\
\hline Outros & 3 & 0,16 & 2 & 0,09 & 3 & 0,16 & 8 & 0,42 & - \\
\hline Total & 1107 & 57,88 & 800 & 41,86 & 5 & 0,26 & 1912 & 100 & $1,38: 1$ \\
\hline
\end{tabular}




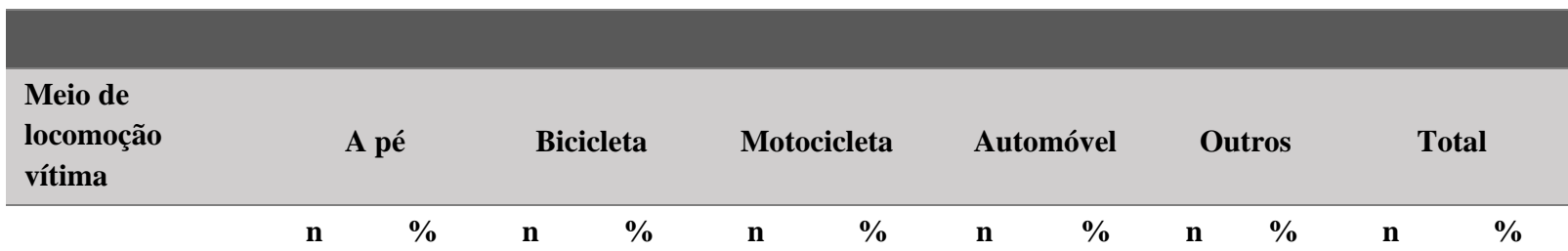

\begin{tabular}{|c|c|c|c|c|c|c|c|c|c|c|c|c|}
\hline $\begin{array}{l}\text { Faixa etária (em } \\
\text { anos) }\end{array}$ & & & & & & & & & & & & \\
\hline 0 a 9 & 10 & 0,52 & 23 & 1,20 & 14 & 0,73 & 18 & 0,94 & 1 & 0,05 & 66 & 3,45 \\
\hline 10 a 19 & 18 & 0,94 & 51 & 2,66 & 127 & 6,64 & 21 & 1,09 & 0 & 0 & 217 & 11,34 \\
\hline 20 a 39 & 38 & 1,98 & 114 & 5,96 & 769 & 40,21 & 97 & 5,07 & 3 & 0,15 & 1021 & 53,39 \\
\hline 40 a 59 & 27 & 1,41 & 79 & 4,13 & 274 & 14,33 & 36 & 1,88 & 2 & 0,10 & 418 & 21,86 \\
\hline 60 ou mais & 19 & 0,99 & 30 & 1,56 & 31 & 1,62 & 20 & 1,04 & 0 & 0 & 100 & 5,23 \\
\hline Ignorado & 7 & 0,36 & 9 & 0,47 & 57 & 2,98 & 17 & 0,88 & 2 & 0,10 & 90 & 4,70 \\
\hline Total & 119 & 6,22 & 306 & 16,00 & 1272 & 66,52 & 207 & 10,82 & 8 & 0,41 & 1912 & 100 \\
\hline
\end{tabular}

Legenda - total de ocorrências; \% - porcentagem do total de ocorrências; M:F - razão entre sexo masculino e feminino. Fonte: elaborada pelo autor.

\section{Discussão}

Os resultados obtidos quanto ao sexo foram equivalentes aos encontrados em muitos outros estudos realizados no país sobre essa temática. Em Dourados, o sexo masculino representou 57,88\% das vítimas de ATT, com uma razão de 1,38 vítimas homens para cada vítima mulher. A literatura atribui esses valores à comportamentos de cunho social e cultural, nos quais os homens tendem a agir de forma inadequada na condução de veículos, utilizando-se por exemplo de alta velocidade, uso de álcool, entre outros fatores de risco para este tipo de acidente, aumentando, desse modo, a exposição a esse tipo de evento (Cabral, Souza \& Lima, 2011; Mendonça, Silva \& Castro, 2017; Biffe et al., 2017; Bastos, Andrade \& Soares, 2005; Miranda \& Sarti, 2011).

Ao analisar os acidentes de trânsito, os homens morrem aproximadamente 3 vezes mais do que as mulheres, entretanto, estudos mais recentes indicam um aumento na participação feminina neste cenário, devido ao crescimento dessa população no mercado de trabalho e na condução dos veículos, o que indiretamente aumenta a exposição a esse tipo de incidente (WHO, 2016; Gawryszewski, 1995; Water, Semenciw \& Mao, 1993).

A distribuição das vítimas por faixa etária evidenciou a predominância em ambos sexos da faixa de 20 a 39 anos e com menor frequência de 0 a 9 anos, com 53,39\% e 3,46\% respectivamente. Pesquisas realizadas entre 1995 e 2015 apresentam resultados que condizem com a presente análise, demonstrando que os jovens adultos, sem dependência do sexo, apresentam maior risco de acometimento por ATT (Mendonça, Silva \& Castro, 2017; Santos et al., 2008; Sallum \& Koizumi, 1999).

Esse resultado não é observado apenas nos estudos brasileiros, uma reunião de dados de países europeus demonstra que os ferimentos por ATT ocorrem majoritariamente em jovens abaixo dos 45 anos, com números que correspondem a $61,0 \%$ do total de mortes por trauma, 62,0\% das hospitalizações e 80,0\% dos atendimentos emergenciais. Bastos et al. (2005), 
Miranda et al. (2011) e Mattox (2000) atribuem esse fato a atitude imprudente e impulsiva particular dessa população, somadas ao uso de álcool e/ou drogas e o desrespeito às regras de trânsito.

Quanto à natureza dos acidentes, as colisões entre veículos automotores foi a causa do incidente em $65 \%$ deles. Assim como nesta pesquisa, as colisões entre veículos automotores aparecem como uma das formas mais comuns de acidentes, visto que os resultados encontrados em Dourados (MS) são correspondentes ao de outras regiões que foram analisadas por Mendonça, Silva \& Castro (2017), Santos et al. (2008), Soares et al. (2012) e Andrade \& Mello-Jorge (2000). Depois das colisões, as quedas ao solo de condutores e passageiros e os atropelamentos a pedestres aparecem em segundo e terceiro lugares, representando $27,5 \%$ e $6,2 \%$ dos incidentes respectivamente. Os prontuários possuem um campo de anotações para os socorristas e na maioria das quedas de condutores e passageiros foram apontados como fatores decisivos para os incidentes a falta de atenção dos motoristas e má conservação das vias da cidade. Nos atropelamentos, o presente estudo demonstrou que a faixa etária mais acometida foi a de 20 a 39 anos, não apontando os idosos como a faixa etária predominante neste tipo de ocorrência, assim como outros estudos (Morena et al., 2016; Alves, 2010).

Conforme a tabela 1 , os motociclistas estiveram presentes em 66,53\% dos acidentes de trânsito atendidos pelo SAMU na cidade. As motocicletas como meio de locomoção de uma vítima de ATT ocuparam o primeiro lugar em diversas outras literaturas brasileiras. Estudos buscaram entender o alto número de motocicletas envolvidas em ATT e alguns fatores foram apontados por Anjos et al. (2007), Marin-Leon et al. (2012) e Felix et al. (2013) chegando ao consenso de que o Brasil é um país em desenvolvimento, no qual o custo de vida é elevado para a maioria da população e neste contexto a motocicleta se apresenta como um veículo barato, ágil, versátil e com baixo custo relativo para sua aquisição e manutenção. Dessa forma, a motocicleta se tornou um veículo popular em números e muito presente na frota brasileira. Em um período de 10 anos (2009 a 2019) na cidade de Dourados (MS), a frota de motocicletas aumentou em torno de 70,12\%, ao mesmo tempo em que a população aumentou cerca de 17,48\% (Brasil, 2020; SEM-MS, 2019).

Nosso estudo tem os finais de semana como os de maior incidência de ATT, com 35,77\% dos incidentes. Ao analisarmos as características dos acidentes com relação ao dia da semana de sua ocorrência, observou-se uma elevação do número de vítimas a partir da sexta-feira, com maior concentração no sábado, cerca de 19,04\% do total. Esses resultados mostraram-se semelhantes aos encontrados na literatura, pois há unanimidade entre alguns autores quanto à maior ocorrência de acidentes de trânsito e de vítimas nos finais de semana (Andrade \& Mello-Jorge, 2000; Minayo, 1994).

Mendonça, Silva \& Castro (2017), Felix et al. (2013) e Minayo (1994) relacionaram a maior ocorrência de acidentes nos finais de semana com a possível ingestão de bebidas alcoólicas pelos motoristas, mais frequente nesses períodos, entretanto, Cabral, Souza \& Lima, (2011) e Soares et al. (2012) relatam que a maior frequência dos acidentes de transporte ocorreu aos domingos, provavelmente em virtude do maior número de eventos comemorativos, entre outros fatores, como por exemplo o consumo de álcool, a ultrapassagem do limite de velocidade e manobras arriscadas.

Em relação ao horário, de acordo com a tabela 2, os ATT estiveram concentrados entre 12:00h e 23:59h, com um total de $62,80 \%$ das ocorrências. Esses números são semelhantes a outros estudos sobre a mesma temática e isso sugere que essa situação pode ter relação com o cansaço, que é maior no final do dia, e também aconteça devido ao elevado fluxo de veículos neste horário. Normalmente o horário da noite é o de maior incidência de vítimas, uma vez que nesse período, geralmente, os acidentes apresentam maior gravidade, em virtude de diversos fatores ligados ao meio ambiente, como a menor visibilidade, e aos usuários da via pública como o excesso de velocidade, desrespeito aos semáforos, uso de álcool e drogas, entre outros (Mendonça, Silva \& Castro, 2017; Santos et al., 2008; Andrade \& Mello-Jorge, 2000).

As equipes do SAMU são multidisciplinares e os veículos possuem todo o suporte e equipamentos para lidar com as várias complicações que advém dos acidentes de trânsito, como grandes hemorragias, paradas respiratórias e outros, e que, se não tratadas corretamente e no tempo certo, podem levar ao óbito da vítima. O atendimento pré-hospitalar rápido e eficiente 
demonstrou sua importância ao preservar a vida, com uma porcentagem de $0,63 \%$ óbitos no local, sendo fundamental para lidar ou até mesmo evitar as complicações provenientes dessas ocorrências.

\section{Conclusão}

O trauma provocado por acidentes de trânsito terrestres merece atenção, devido a sua prevalência e gravidade, especialmente em relação ao planejamento de ações preventivas, com o objetivo de controlar sua ocorrência. O reconhecimento e sistematização das características epidemiológicas da população atingida por um determinado agravo, a epidemiologia desses incidentes ao longo do tempo e a determinação dos grupos mais expostos possibilitam a criação e implantação de estratégias de prevenção que podem diminuir os riscos e as consequências deste tipo de incidente.

O estudo dos ATT a partir das fichas do SAMU apresentou ótimos resultados, demonstrando que esses dados possuem grande valor como gerador de informação para a vigilância de saúde. As limitações do estudo relacionam-se à cobertura populacional, visto que na cidade de Dourados-MS, o Corpo de Bombeiros Militar presta atendimento de emergência a parcela da população, além de vítimas que não buscaram atendimento ao SAMU e também não foi possível sua inclusão na amostra. Apesar dessas limitações, acredita-se que este estudo conseguiu traçar o perfil das vítimas, gerando informações relevantes acerca das variáveis atendidas como sexo, idade, dia da ocorrência, natureza do incidente, entre outras, que definiram grupos e situações de risco, oferecendo dessa forma pontos de referência confiáveis para o planejamento de ações de prevenção a saúde.

Em estudos retrospectivos como este, o levantamento de dados é uma etapa difícil e muito importante, visto que dela serão colhidas todas as informações necessárias para realização do trabalho. Recomenda-se para trabalhos futuros a maior abrangência possível em relação a coleta de dados, com uso de diferentes fontes e cruzamento de dados, em busca de uma base sólida para análise e escrita. Além disso, recomendamos a realização de novos estudos relacionados ao tema para que os dados sempre estejam atualizados na literatura, devido a importância dos mesmos no planejamento de ações.

As ações de prevenção a agravos devem ser baseadas em perfis epidemiológicos, sempre que possível respeitando as características regionais e temporais do local, em busca dos melhores resultados dessas ações. Diante do exposto no presente estudo, são recomendadas intervenções com o objetivo de prevenir os acidentes de trânsito, com a priorização de investimentos em iluminação, sinalização viária e manutenção geral das vias públicas. Além disso, ações de educação são importantes, desde cedo na conscientização dos jovens futuros condutores, bem como dos condutores mais experientes.

\section{Agradecimentos}

Os autores agradecem ao Serviço de Atendimento Móvel de Urgência (SAMU - Regional Dourados), especialmente as pessoas de Sandro Barreto dos Santos, Ronaldo Marques Sobrinho e Letícia Martinelli Barbosa da Silva pela colaboração e todo auxilio necessário no processo de obtenção dos dados.

\section{Referências}

Alves, E.F. (2010). Características dos Acidentes de Trânsito com Vítimas de Atropelamento no Município de Maringá-Pr, 2005-2008. Saúde e pesquisa, v. 3, n. 1, 2010. https://periodicos.unicesumar.edu.br/index.php/saudpesq/article/view/1224

Andrade, S.M. \& Mello-Jorge, M.H.P. (2000). Características das vítimas por acidentes de transporte terrestre em município da Região Sul do Brasil. Rev. Saúde Pública. Abr; 34(2): 149-156 https://doi.org/10.1590/S0034-89102000000200008

Anjos, K.C., Evangelista, M.R.B., Silva, J.S. \& Zumiotti, A.V. (2007) Paciente vítima de violência no trânsito: análise do perfil socioeconômico, características do acidente e intervenção do serviço social na emergência. Acta Ortopédica Brasileira. 2007; 15(5):262-266. https://doi.org/10.1590/S141378522007000500006 
Araújo, D. C., Almeida, C.P., Santana, L.R.P., Santos, A.D., Lima, S.V.M.A. et al. (2021). Predicting factors and quality of life of trauma victims for traffic accidents. Research, Society and Development, [S. l.], v. 10, n. 5, p. e0410514576, 2021. https://rsdjournal.org/index.php/rsd/article/view/14576

Bailey, T.C. (2001). Métodos estatísticos espaciais em saúde. Cad Saúde Pública 2001; 17(5): 1083-98. https://doi.org/10.1590/S0102-311X2001000500011

Barros, A.J.D., Amaral, R.L, Oliveira, M.S.B, Lima, S.C. \& Gonçalves, E.V. (2003). Acidentes de trânsito com vítimas: sub-registro, caracterização e letalidade. Cad. Saúde Pública. 2003 Ago; 19(4): 979-986. https://doi.org/10.1590/S0102-311X2003000400021

Bastos, Y.G.L., Andrade, S.M. \& Soares, D.A. (2005). Características dos acidentes de trânsito e das vítimas atendidas em serviço pré-hospitalar em cidade do Sul do Brasil, 1997/2000. Cad. Saúde Pública. 2005 Jun; 21(3): 815-822. https://doi.org/10.1590/S0102-311X2005000300015

Biffe, C.R.F, Harada, A., Bacco, A.B, Coelho, C.S, Baccarelli, J.L.F. et al. (2017). Perfil epidemiológico dos acidentes de trânsito em Marília, São Paulo, 2012. Epidemiol. Serv. Saúde. 2017 Jun; 26( 2 ): 389-398. https://doi.org/10.5123/S1679-49742017000200016

Brasil. Ministério da Saúde. (2006). Secretária de Atenção à Saúde. Coordenação-Geral de Urgência e Emergência. Política nacional de atenção às urgências. Série E. Legislação de Saúde. https://bvsms.saude.gov.br/bvs/publicacoes/politica_nacional_atencao_urgencias_3ed.pdf

Brasil. Ministério da Saúde. (2006). Secretária de Atenção à Saúde. Coordenação-Geral de Urgência e Emergência. Política nacional de atenção às urgências. Série E. Legislação de Saúde. https://bvsms.saude.gov.br/bvs/publicacoes/politica_nacional_atencao_urgencias_3ed.pdf

Brasil. Ministério da Saúde. (2018). DATASUS. Sistema de informações de mortalidade - SIM http://tabnet.datasus.gov.br/cgi/tabcgi.exe?sim/cnv/obt10uf.def

Brasil. Ministério dos Transportes. (2020). Departamento Nacional de Trânsito. Estatísticas das frotas de veículos por munícipio brasileiro https://www.gov.br/infraestrutura/pt-br/assuntos/transito/conteudo-denatran/estatisticas-frota-de-veiculos-denatran

Cabral, A.P.S., Souza, W.V.D. \& Lima, M.L.C.D. (2011). Serviço de atendimento móvel de urgência: um observatório dos acidentes de transportes terrestre em nível local. Revista Brasileira de Epidemiologia, 14(1), 03-14. https://doi.org/10.1590/S1415-790X2011000100001

Cerqueira, G.L.C. (2015). Consumo de álcool e outras drogas por jovens condutores. Revista Psicologia.pt. 2015 out; $1646-6977$. https://www.psicologia.pt/artigos/textos/A0919.pdf

Derenzo, N., Miranda, D.J.C., Silva, S.L.P., Neves, I.F., Mendonça, R.R. et al. (2021). Perfil do atendimento de urgência e emergência em uma base do

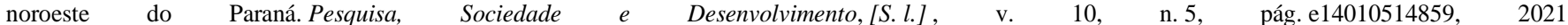
https://rsdjournal.org/index.php/rsd/article/view/14859

Dourados. Portal da Prefeitura de Dourados. (2020). Conheça a cidade de Dourados. http;//dourados.ms.gov.br/index.php/cidade-de-dourados/

Duarte, S.J.H, Nardes, R.P.M.A., Pena, S.B., Mendez, R.B.R. \& Candido, M.C.F.S. (2013). Vítimas de acidente motociclístico atendidas pelo serviço de atendimento móvel de urgência em Campo Grande, MS. Enfermagem em Foco, [S.1.], v. 4, n. 2, maio 2013. ISSN 2357-707X. https://doi.org/10.21675/2357707X.2013.v4.n2.530

Felix, N.R., Oliveira, S.R., Cunha, N.A. \& Schirmer, C. (2013). Caracterização das vítimas de acidente motociclistico atendidas pelo serviço de atendimento pré-hospitalar. Revista Eletrônica Gestão \& Saúde 2013; 04(04): 1399-411. https://dialnet.unirioja.es/servlet/articulo?codigo=5557496

Gawryszewski, V.P. (1995). A mortalidade por causas externas no Município de São Paulo, 1991. Dissertação de Mestrado. Faculdade de Saúde Pública da USP. https://pesquisa.bvsalud.org/ripsa/resource/pt/lil-162245

IPEA. Instituto de Pesquisa Econômica Aplicada, (2020). Impactos Sociais e Econômicos dos Acidentes de Trânsito nas Rodovias Brasileiras. https://www.ipea.gov.br/atlasviolencia/arquivos/artigos/7018-td2565.pdf

Ludke, M. \& Andre, M. E . D. A. (2013). Pesquisas em educação: uma abordagem qualitativa. São Paulo: E.P.U.

Marín-Leon, L., Belon, A.P., Barros, M.B.A., Almeida, S.D.M. \& Restitutti, M.C. (2012). Tendência dos acidentes de trânsito em Campinas, São Paulo, Brasil: importância crescente dos motociclistas. Caderno de Saúde Púbica. 2012; 28(1):39-51. http://dx.doi.org/10.1590/S0102-311X2012000100005

Marques, T.O., Melo, L.D., Taroco, F.E., Duarte, R.M.L, Lima, H.D. (2021). Serviços de Atendimento Móvel de Urgência (Mecs): uma revisão integrativa. Pesquisa, Sociedade e Desenvolvimento , [S. l.] , v. 10, n. 2, pág. e38310212522, 2021. https://rsdjournal.org/index.php/rsd/article/view/12522

Mattox, K.L., Feliciano, D.V., \& Moore, E.E. (2000). Trauma. New York: McGraw-Hill.

Mendonça, M.F.S., Silva, A.P.S.C. \& Castro, C.C.L. (2017). Análise espacial dos acidentes de trânsito urbano atendidos pelo Serviço de Atendimento Móvel de Urgência: um recorte no espaço e no tempo. Rev. bras. epidemiol. 2017 Dez; 20(4): 727-741. https://doi.org/10.1590/1980-5497201700040014

Minayo, M.C.S. (1994). Violência social sob a perspectiva da saúde pública. Cad Saúde Pública 1994; 10 Suppl 1:7-18. https://doi.org/10.1590/S0102$311 \mathrm{X} 1994000500002$

Miranda, A.L. \& Sarti, E.C.F.B. (2011). Consumo de bebidas alcoólicas e os acidentes de trânsito: o impacto da homologação da lei seca em Campo GrandeMS. Ensaios Cienc, Cienc Biol Agrar Saúde 2011; 15(6): 155-71. https://doi.org/10.17921/1415-6938.2011v15n6p\%25p

Moreno, N.T.S., Dalmas, J.C. \& Martins, E.A.P. (2016). Atropelamentos: análise epidemiológica dos últimos quatro anos. Ciênc. cuid. saúde, p. 693-700, 2016. https://doi.org/10.4025/cienccuidsaude.v15i3.34558

Moysés, S.J. (2012). Determinação sociocultural dos Acidentes de Transporte Terrestre (ATT). Ciênc. Saúde coletiva. 2012 Set; 17(9): 2241-2243. https://doi.org/10.1590/S1413-81232012000900005 
MS. Mato Grosso do Sul. (2019). Secretaria Estadual de Saúde - SES. Diário Oficial Eletrônico $n^{\circ} 9.937$ de 8 de julho de 2019. Dispõe sobre o plano de ação regional da rede de atenção às urgências e emergências do estado de mato grosso do sul. http://www.as.saude.ms.gov.br/wpcontent/uploads/2019/11/Resolu\%C3\%A7\%C3\%A3o-n\%C2\%BA-74.CIB_.SES-de-27.06.2019-Integrantes-Comiss\%C3\%A3o-Estadual-de-

Farmacoterapia.pdf

Nolasco, T.R, Andrade, S.M. \& Silva, B.A.K. (2016). Capacidade funcional de vítimas de acidentes de trânsito em Campo Grande, Mato Grosso do Sul. Ensaios Cienc, Cienc Biol Agrar Saúde 2016; 20(2): 104-10. http://dx.doi.org/10.17921/1415-6938.2016v20n2p104-110

Sallum, A.M.C. \& Koizumi, M.S. (1999). Natureza e gravidade das lesões em vítimas de acidente de trânsito de veículo a motor. Rev. esc. enferm. USP. 1999 jun.; 33(2): 157-164. https://doi.org/10.1590/S0080-62341999000200007

Santos, A.M.R., Moura, M.E.B., Nunes, B.M.V.T., Leal, C.F.S. \& Teles, J.B.M. (2008). Perfil das vítimas de trauma por acidente de moto atendidas em um serviço público de emergência. Cad. Saúde Pública. 2008 Ago; 24 (8): 1927-1938. https://doi.org/10.1590/S0102-311X2008000800021

Santos, S.M.J., Souza, M.A., Rocha, F.L., Souza, V.P., Muniz, M.A.S. et al. (2016). Caracterização dos fatores de risco para acidentes de trânsito em vítima atendidas pelo serviço móvel de urgência. Revista de Enfermagem UFPE Online. Recife, 10(10):3819-24, out., 2016 https://doi.org/10.5205/1981-8963v10i10a11448p3819-3824-2016

SBAIT. Sociedade Brasileira de Atendimento Integral ao Traumatizado. (2005). Projeto Trauma 2005-2025. http://files.lateme.webnode.com.br/200000038db7e4dc78b/Projeto\%20Trauma\%202005-2025.pdf

Silva, N.C. \& Nogueira, L.T. (2012). Avaliação de indicadores operacionais de um serviço de atendimento móvel de urgência. Cogitare Enfermagem, 17(3). http://dx.doi.org/10.5380/ce.v17i3.29287

Soares, R.A.S., Pereira, A.P.J.T., Moraes, R.M. \& Vianna, R.P.T. (2012). Caracterização das vítimas de acidentes de trânsito atendidas pelo Serviço de Atendimento Móvel de Urgência (SAMU) no Município de João Pessoa, Estado da Paraíba, Brasil, em 2010. Epidemiol. Serv. Saúde. 2012 Dez]; 21(4): 589600. http://dx.doi.org/10.5123/S1679-49742012000400008

Sousa, P.G., Nogueira, T. A., Meza, R. O.; Oliveira, V. A., \& Abreu, M.H.T. (2021). Spatial analysis of fatal victims of motorcycle traffic accidents in Teresina - Piauí. Research, Society and Development, [S. l.], v. 9, n. 10, p. e9549109506, 2020. https://rsdjournal.org/index.php/rsd/article/view/9506

Tobase, L., Tomazini, E.A.S., Teodoro, S.V., Piza, N.R.G., \& Peres, H.H.C. (2012). Ensino à distância na educação permanente em Urgência e Emergência. Journal of Health Informatics. http://www.jhi-sbis.saude.ws/ojs-jhi/index.php/jhi-sbis/article/view/242/131

Vidmar, G. R., Lohmann, P. M., Silva, G.L., Costa, A.E.K.; \& Marchese, C. (2021). Scene time in trauma care at the Emergency Mobile Care Service (EMCS) in a city in the interior of the State of Rio Grande do Sul from 2012 to 2019. Research, Society and Development, [S. l.], v. 9, n. 8, p. e25985162, 2020. https://rsdjournal.org/index.php/rsd/article/view/5162

Waters, C., Gibbons, L., Semenciw, R. \& Mao, Y. (1993). Motor vehicle traffic accidents in Canada, 1978-87 by time of occurrence. Rev Can Santé Publique 1993;84:58-9. https://pubmed.ncbi.nlm.nih.gov/8500060/

WHO. World Health Organization. (2016). Road traffic injuries. Fact sheet. https://www.who.int/news-room/fact-sheets/detail/road-traffic-injuries

WHO. World Health Organization. (2018). Global status report on road safety 2018. https://www.who.int/publications/i/item/9789241565684 